\begin{tabular}{|c|c|}
\hline Title & A nalysis of magnetic shielding effect of lay ered shields based on homogenization \\
\hline Author(s) & Waki, Hiroshi; Igarashi, Hajime; Honma, Toshihisa \\
\hline Citation & $\begin{array}{l}\text { IEEE transactions on magnetics, 42(4), } 847-850 \\
\text { https://doi.org/10.1109/ MA G.2006.872480 }\end{array}$ \\
\hline Issue Date & $2006-04$ \\
\hline Doc URL & http:/hdl .handle.net/2115/8518 \\
\hline Rights & $\begin{array}{l}\text { (c) } 2006 \text { IEEE. Personal use of this material is permitted. However, permission to reprint/republish this material for } \\
\text { advertising or promotional purposes or for creating new collective works for resale or redistribution to servers or lists, } \\
\text { or to reuse any copyrighted component of this work in other works must be obtained from the IEEE }\end{array}$ \\
\hline Type & article \\
\hline File Information & 01608339.pdf \\
\hline
\end{tabular}

Instructions for use 


\title{
Analysis of Magnetic Shielding Effect of Layered Shields Based on Homogenization
}

\author{
Hiroshi Waki, Hajime Igarashi, and Toshihisa Honma \\ Graduate School of Information Science and Technology, Hokkaido University, Hokkaido, Japan
}

\begin{abstract}
This paper describes a nonlinear analysis of magnetic field based on homogenization. The analysis of a magnetic field is time-consuming when the problem includes magnetic substance with fine structure. Simplification of the fine structure by homogenization makes it possible to analyze them efficiently. The authors have introduced a homogenization method to estimate effective permeability of magnetic composite structure for a static field. This method can be applied not only for linear problems but also nonlinear ones. In this paper, the magnetic shielding effect of layered nonlinear material is analyzed by using the homogenization method, and the applicability of this method is discussed.
\end{abstract}

Index Terms-Effective permeability, homogenization, magnetic shielding, nonlinear analysis.

\section{INTRODUCTION}

$\mathbf{M}$ AGNETIC fields generated by direct current (DC) power lines such as feeders along electric railways sometimes interfere with electronic instruments, for example, cathode-ray tubes and electron microscopes, and so on. Magnetic shields composed of steel rods and steel plates are expected to attenuate the magnetic fields. Numerical analyses of the magnetic fields are necessary to study the optimum arrangement of the steel rods and the steel plates for magnetic shielding. The magnetic shielding effects can be analyzed in theory by using the finite element method (FEM), etc. However, the analyses become time-consuming, because the magnetic shields have a fine structure.

Simplification of the structure of the magnetic shields by homogenization makes it possible to analyze them efficiently [1], [2]. A lot of homogenizing methods have been introduced in order to estimate the effective material parameters of composite materials and mixtures so far [3]. The homogenization has also been studied in fields of remote sensing [4], [5]. The most wellknown and standard rules employs the Maxwell-Garnett formula [6], [7]. In recent years, Sareni et al. have introduced a homogenization method in which the effective permittivity is obtained on the basis of the potential [8].

The authors have introduced a homogenization method to estimate the effective permeability of the composite structure [9]. In this method, the structure of the magnetic shields is assumed to be periodic, and the unit cell is defined, in which the effective permeability is determined from consistency of magnetic energy. This method is more accurate than the conventional models [9], and it can be applied not only for linear problems but also nonlinear ones [10].

In this paper, the magnetic shielding effect of the layered nonlinear material is analyzed by using the homogenization method, and then the applicability of this method is discussed.

Digital Object Identifier 10.1109/TMAG.2006.872480

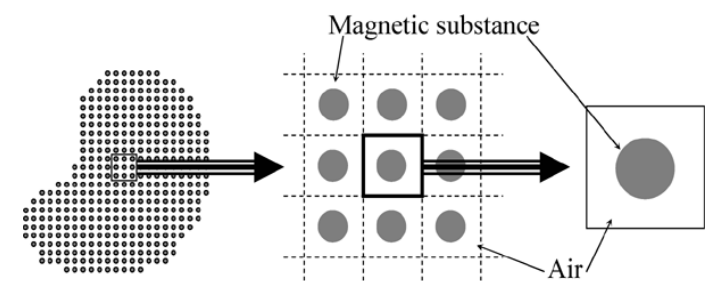

Original

Unit cell
Fig. 1. Definition of unit cell.

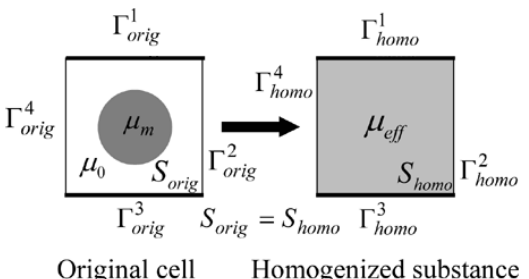

Fig. 2. Homogenization of cell. Both cells are immersed in equivalent field.

\section{Nonlinear EfFective PermeabiLITy}

Fig. 1 shows a two-dimensional composite material, which includes cylindrical steel rods with isotropic magnetism. In the homogenization method, the structure of the magnetic composite materials is assumed to be periodic, and the unit cell, which is the minimum volume to represent the overall statistics, is defined. Here, it is assumed that one cylinder is located in the center of the cell, and the cell has also structural isotropy. The unit cell is regarded as a homogeneous magnetic substance with the effective permeability as shown in Fig. 2. The effective permeability is defined on the basis of magnetic energy balance in the unit cell: it is assumed that the original cell and the homogenized cell include equivalent magnetic energy when both unit cells are immersed in equivalent magnetic field.

In an actual estimation, the solution of the Laplace equation is computed by FEM. In this analysis, vector potentials are unknowns, and assuming the applied field is unidirectional at least in the cell, say, in the $x$-direction, the boundary conditions are set as

$$
A=A_{1} \quad \text { on } \quad \Gamma_{\text {orig }}^{1}, \Gamma_{\text {homo }}^{1}
$$




$$
\begin{aligned}
A & =A_{3} \text { on } \Gamma_{\text {orig }}^{3}, \Gamma_{\text {homo }}^{3} \\
\frac{\partial A}{\partial n} & =0 \text { on } \Gamma_{\text {orig }}^{2}, \Gamma_{\text {orig }}^{4}, \Gamma_{\text {homo }}^{2} \text { and } \Gamma_{\text {homo }}^{4}
\end{aligned}
$$

where $A$ represents the vector potential, $n$ the normal vector to $\Gamma_{\text {orig }}^{\iota}$ or $\Gamma_{\text {homo }}^{\iota}$. The boundary conditions are equally set to both cells, and the applied field $\mathbf{B}_{\text {ap }}$ is determined, which is equal to the magnetic flux density in the homogenized cell $\mathbf{B}_{\text {homo. These }}$ vectors have only $x$-components. Thus, for simplicity, the index $x$ will be omitted hereafter. When the homogenization method is applied to nonlinear magnetic materials, the magnetic field in the unit cell is evaluated by the Newton-Raphson technique.

The magnetic energy in the original cell is represented as follows:

$$
U_{\text {orig }}\left(B_{\text {ap }}\right)=\int_{S_{\text {orig }}} \int_{\mathbf{B}_{\text {orig }}} \mathbf{H}_{\text {orig }} \cdot d \mathbf{B}_{\text {orig }} d S_{\text {orig }}
$$

where $\mathbf{B}_{\text {orig }}$ represents the magnetic flux density, $\mathbf{H}_{\text {orig }}$ the magnetic field, $S_{\text {orig }}$ the area of the original cell. The magnetic energy in the homogenized cell is represented as follows:

$$
U_{\text {homo }}\left(B_{\text {ap }}\right)=S_{\text {homo }} \int_{B_{\text {homo }}} H_{\text {homo }} d B_{\text {homo }}
$$

where $H_{\text {homo }}$ represents the magnetic field, $S_{\text {homo }}$ the area of the homogenized cell. In the homogenization method, it is assumed that the magnetic energy in both cells is equivalent, therefore a relation of the energy balance

$$
U_{\text {orig }}\left(B_{\text {ap }}\right)=U_{\text {homo }}\left(B_{\text {ap }}\right)
$$

is organized. For the linear problems, from (4), (5), and (6), the effective permeability is given as follows:

$$
\mu_{\mathrm{eff}}=\frac{\int_{S_{\text {homo }}}\left(B_{\text {homo }}\right)^{2} d S_{\text {homo }}}{\int_{S_{\text {orig }}} \mathbf{B}_{\text {orig }} \cdot \mathbf{H}_{\text {orig }} d S_{\text {orig }}}
$$

which is held for any applied field [9].

For the nonlinear problem, the applied field is increased in the following form:

$$
B_{\mathrm{ap}}^{j}=\left\{\begin{array}{ll}
0 & (j=0) \\
B_{\mathrm{ap}}^{j-1}+\Delta B_{\mathrm{ap}} & (j=1,2, \ldots)
\end{array} .\right.
$$

When the $j$ th magnetic field $B_{\text {ap }}^{j}$, which is equal to $B_{\text {homo }}^{j}$, is applied to the cells, the magnetic energy in the homogenized cell is equal to $U_{\text {homo }}^{j}$ as shown in Fig. 3, which is equal to summation of the magnetic energy $\Delta U_{\text {homo }}^{j}$. Thus, the $j$ th magnetic energy in the homogenized cell are represented as follows:

$$
U_{\text {homo }}^{j}=\sum_{j} \frac{H_{\text {homo }}^{j-1}+H_{\text {homo }}^{j}}{2} \Delta B_{\text {homo }}^{j} S_{\text {homo }} .
$$

The magnetic energy in the original cell is also obtained by this approach as follows:

$$
\begin{aligned}
U_{\text {orig }}^{j} & =\sum_{e} \sum_{j} \frac{\mathbf{H}_{\text {orig }}^{e, j-1}+\mathbf{H}_{\text {orig }}^{e, j}}{2} \cdot \Delta \mathbf{B}_{\text {orig }}^{e, j} S_{\text {orig }}^{e} \\
\Delta \mathbf{B}_{\text {orig }}^{e, j} & =\mathbf{B}_{\text {orig }}^{e, j}-\mathbf{B}_{\text {orig }}^{e, j-1}
\end{aligned}
$$

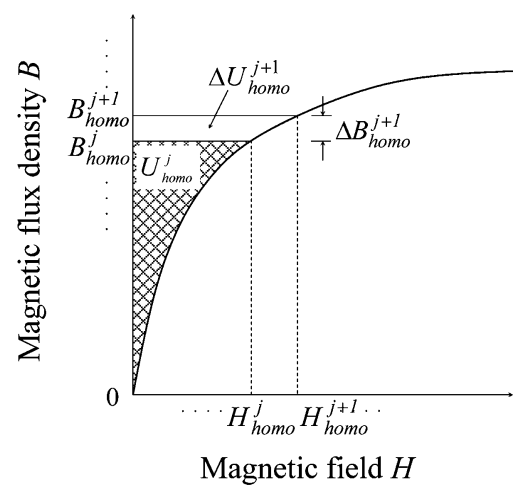

Fig. 3. Estimation of magnetic energy in homogenized cell. This process is applied for nonlinear problems.

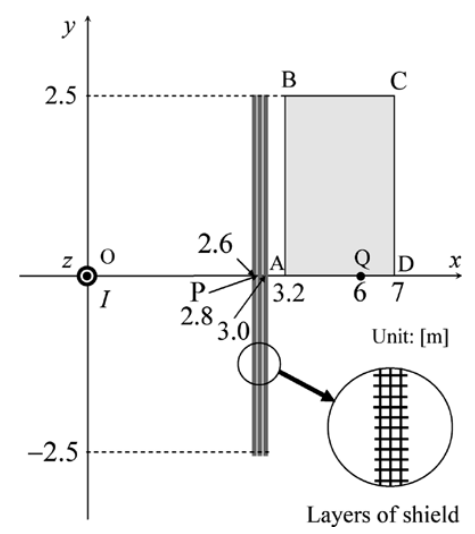

Fig. 4. Numerical example. The applied field is generated by direct current $I$. The error $E_{\mathrm{ABCD}}$ is evaluated on the area $\mathrm{ABCD}$. The attenuation $\mathrm{A}_{\mathrm{Q}}$ is evaluated at $\mathrm{Q}$.
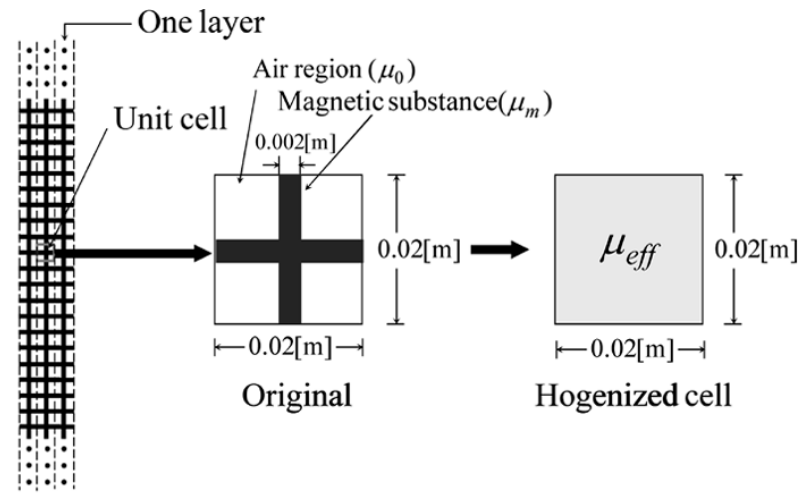

Hogenized cell

Layers of shield

Fig. 5. Unit cell of layered shield.

where $e$ represents the element in the original cell. From (6), (8), (9), (10) and (11), the magnetic field in the homogenized cell is given as follows:

$$
H_{\mathrm{homo}}^{j}=\frac{\sum_{e}\left(\mathbf{H}_{\mathrm{orig}}^{e, j-1}+\mathbf{H}_{\mathrm{orig}}^{e, j}\right) \cdot \Delta \mathbf{B}_{\mathrm{orig}}^{e, j} S_{\mathrm{orig}}^{e}}{\Delta B_{\mathrm{homo}}^{j} S_{\mathrm{homo}}}-H_{\mathrm{homo}}^{j-1}
$$




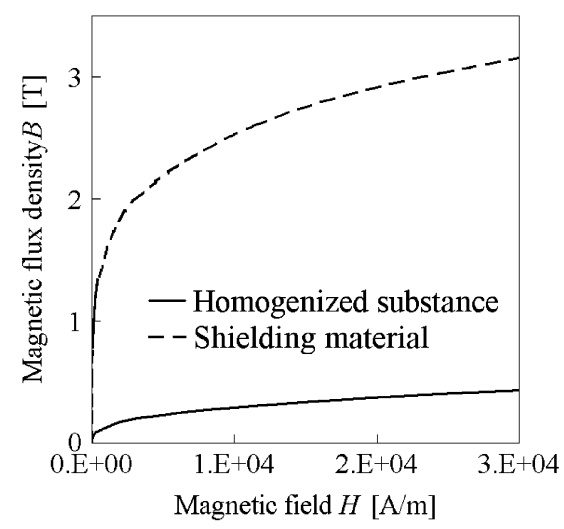

Fig. 6. $B-H$ property of shielding material and homogenized substance in nonlinear problem.

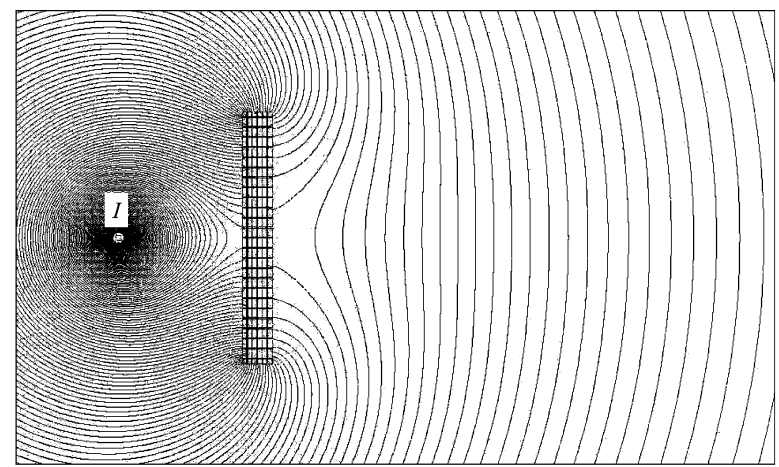

(a)

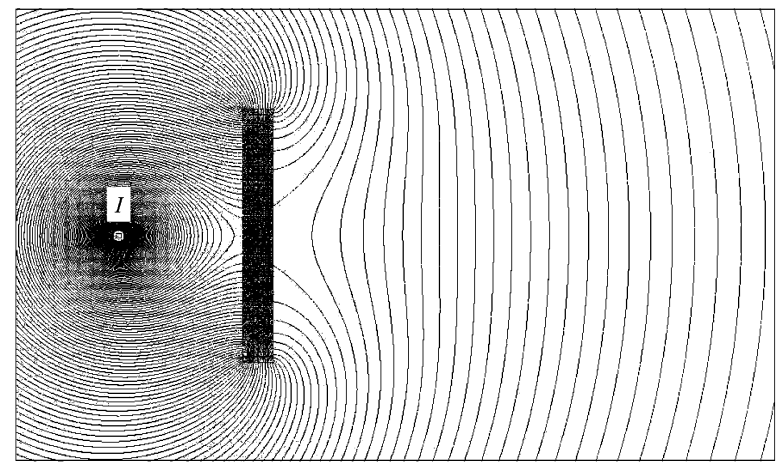

(b)

Fig. 7. Magnetic flux line in nonlinear problem. The layered shield is composed of 3 layers. The magnetic flux density $0.01 \mathrm{~T}$ is applied to the shield. (a) Layered shield. (b) Homogenized substance.

The $B-H$ property of the homogenized substance can be obtained from (12), and then the effective permeability is given by as follows:

$$
\mu_{\mathrm{eff}}\left(B_{\mathrm{ap}}^{j}\right)=\frac{\Delta B_{\text {homo }}^{j}}{\Delta H_{\text {homo }}^{j}} .
$$

\section{ANALYSIS OF MAGNETIC SHIELDING EFFECT}

The magnetic shielding effect of the layered substance is analyzed by using the homogenization method. In this example, the magnetic shield is in layers along the $z$-axis as shown in Fig. 4, and the magnetic field generated by the DC current $I$ is applied to the shield. Fig. 5 shows the structure of the magnetic shield

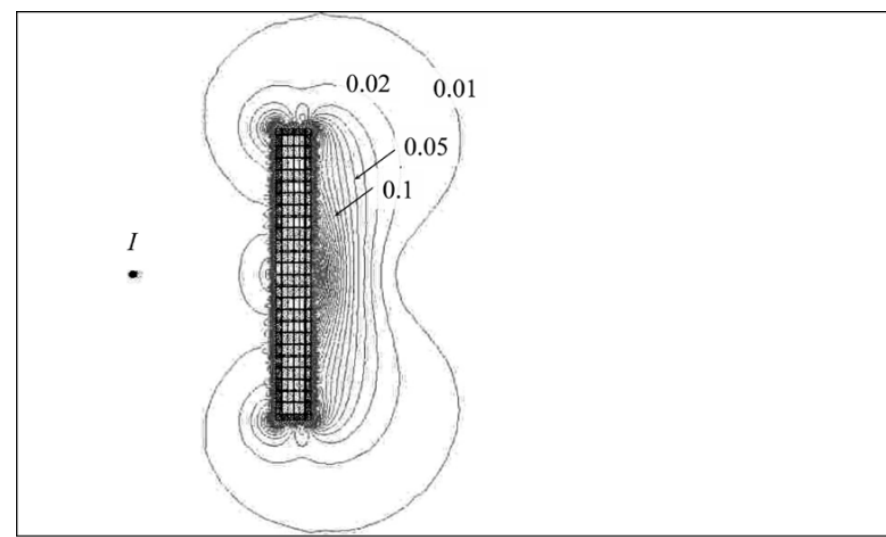

(a)

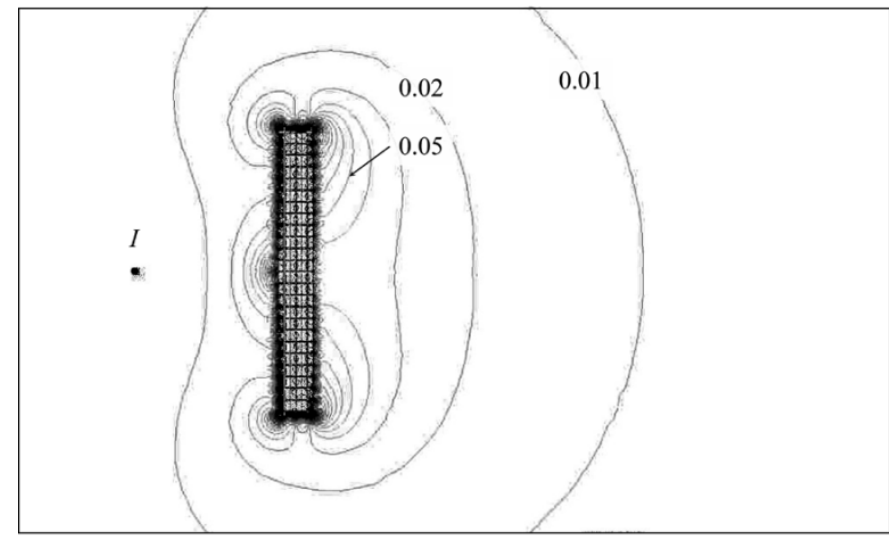

(b)

Fig. 8. Distribution of error. These errors are evaluated by the equivalent condition of Fig. 7. (a) Nonlinear problem. (b) Linear problem.

and the unit cell. This shield is homogenized by the above-mentioned method. The homogenized substance is also applied to this example as well as the original material.

In this example, the magnetic field for this 2-D problem is analyzed by using FEM, and then the error on $\mathrm{ABCD}$ as shown in Fig. 4. The error is here defined as

$$
E=\sqrt{\sum_{e}\left|\mathbf{B}_{\text {homo }}^{e}-\mathbf{B}_{\text {orig }}^{e}\right|^{2} S^{e} / \sum_{e}\left|\mathbf{B}_{\text {orig }}^{e}\right|^{2} S^{e}}
$$

where $S^{e}$ represents the area of the element $e, \mathbf{B}_{\text {orig }}^{e}$ the magnetic field in case of the original material, $\mathbf{B}_{\text {homo }}^{e}$ the magnetic field in case of the homogenized substance.

The shielding effect is evaluated by attenuation of the magnetic field defined as

$$
A=20 \log \left(\left|\mathbf{B}_{s}\right| /\left|\mathbf{B}_{0}\right|\right)
$$

where $\mathbf{B}_{\mathrm{s}}$ represents the magnetic field affected by the magnetic shield, $\mathbf{B}_{0}$ the magnetic field in the case of no shield.

\section{RESUlT AND DISCUSSION}

Fig. 6 shows the $B-H$ property of the shielding material and the homogenized substance. The curve of the homogenized substance is evaluated by the nonlinear approach from the magnetic property of the shielding material. To compare with a linear problem, the effective permeability in case of a linear magnetic material is also evaluated. When the permeability of the 


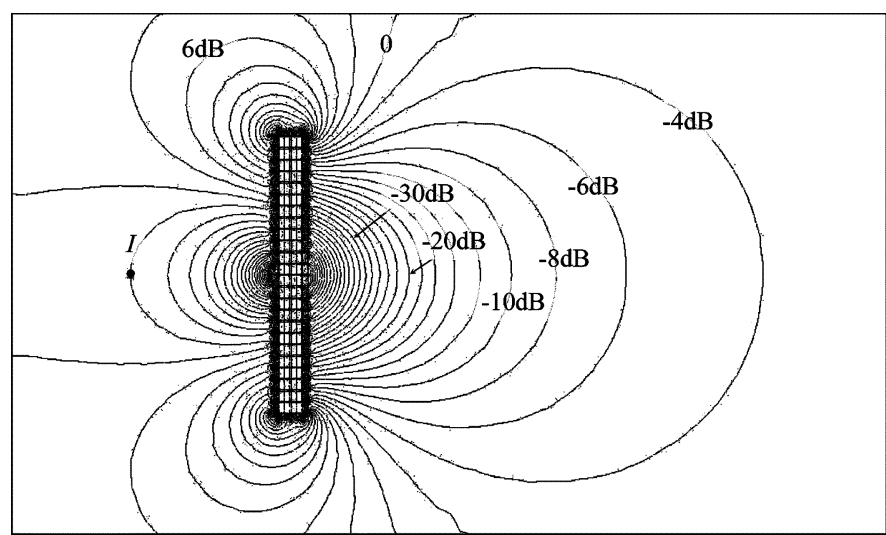

Fig. 9. Attenuation $A$ of magnetic field by shield in nonlinear problem. This attenuation is evaluated by the equivalent condition of Fig. 7.

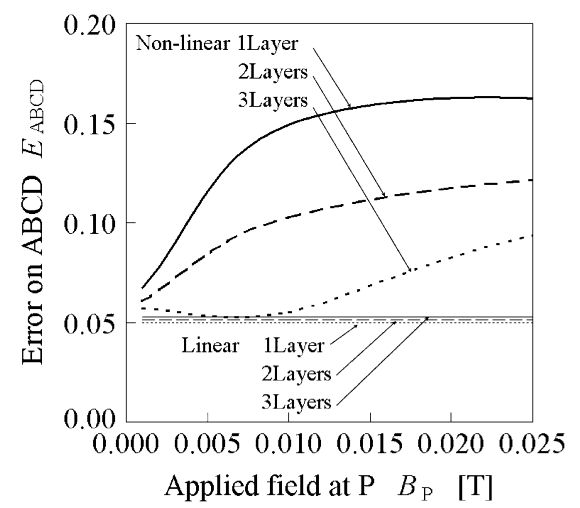

Fig. 10. Error versus applied field.

shielding material is set to $1000 \mu_{0}$, then the linear effective permeability is estimated to be $103.80 \mu_{0}$ by (7).

Fig. 7 shows the magnetic flux line affected by the shield. The distribution in the case of homogenized substance is nearly equal to that in case of the original. Fig. 8 shows the error of the magnetic field. In the nonlinear case, the error near the shield is relative large. However, the overall accuracy seems satisfactory.

Fig. 9 shows the distribution of the attenuation $A$. Near the center of the shield, the magnetic field is attenuated on the order of several ten decibels. The attenuation increases with the distance from the shield.

Fig. 10 shows the error versus the applied field. Under low field, the errors are nearly equal to those of the linear problem. However, the errors tend to increase with the applied field. In the homogenization method, the nonlinear magnetic property is evaluated by integral of the magnetic energy. In this process, inadequate $\Delta \mathbf{B}_{\text {ap }}$ would cause deterioration in the accuracy.

Fig. 11 shows the attenuation at $\mathrm{Q}$. The attenuation decreases with increasing of the applied field, because the permeability decreases due to the nonlinear effect.

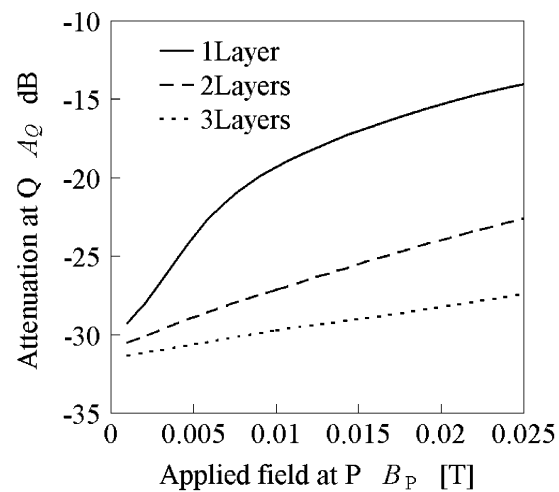

Fig. 11. Attenuation versus applied field in nonlinear problem.

\section{CONCLUSION}

The magnetic shielding effect of the layered nonlinear material can be analyzed by using the homogenization method. Under low field, this method is accurate as well as the linear problem. The error tends to increase with the applied field. However, the overall accuracy seems satisfactory. It is future work to apply the homogenization method to a cell having structural anisotropy.

\section{REFERENCES}

[1] H. Waki, H. Igarashi, and T. Honma, "Modeling of reinforced concrete structures for analysis of environmental magnetic fields," Comput. Eng. I, JASCOME, pp. 117-124, 2004

[2] — "An analysis of magnetic shielding against DC power lines based on homogenization," COMPEL, vol. 24, no. 2, pp. 566-580, Feb. 2005.

[3] E. Tuncer, Y. V. Serdyuk, and S. M. Gubanski, "Dielectric mixtures: Electrical properties and modeling," IEEE Trans. Dielectr. Electr. Insul., vol. 9, no. 5, pp. 809-828, Oct. 2002.

[4] A. H. Sihvola and J. A. Kong, "Effective permittivity of dielectric mixtures," IEEE Trans. Geosci. Remote Sens., vol. 26, no. 4, pp. 420-429, Jul. 1988.

[5] K. K. Karkkainen, A. H. Sihvola, and K. I. Nikoskinen:, "Effective permeability of mixtures: numerical validation by FDTD method," IEEE Trans. Geosci. Remote Sens., vol. 38, no. 3, pp. 1303-1308, May 2000.

[6] J. C. M. Garnett, "Colors in material glasses and metal films," Trans. $R$. Soc., vol. 53, pp. 385-420, 1904.

[7] A. Sihvola, "Electromagnetic mixing formulas and applications," IEE Electromagnetic Waves Series 47, pp. 63-68, 1999.

[8] B. Sareni, L. Krahenbuhl, A. Beroual, A. Nicolas, and C. Brosseau, "A boundary integral equation method for the calculation of the effective permittivity of periodic composites," IEEE Trans. Magn., vol. 33, no. 2, pp. 1580-1583, Mar. 1997.

[9] H. Waki, H. Igarashi, and T. Honma, "Estimation of effective permeability of magnetic composite materials," IEEE Trans. Magn., vol. 41, no. 5, pp. 1520-1523, May 2005.

[10] — - "Estimation of nonlinear effective permeability of magnetic materials with fine structure," Physica B, vol. 372, pp. 383-387, Feb. 2006.

Manuscript received June 20, 2005 (e-mail: waki@em-si.eng.hokudai.ac.jp). 